Anal ysi s of hi gher har moni cs on bi di rect i onal heat pul se propagat i on experi ment i $n$ hel i cal and tokamak pl asmas

\begin{tabular}{|l|l|}
\hline $\begin{array}{l}\text { jour nal or } \\
\text { publ i cat i on } \mathrm{titl} \text { e }\end{array}$ & Nucl ear Fusi on \\
\hline vol une & 57 \\
\hline number & 7 \\
\hline page range & 076013 \\
\hline year & 2017- 05- 17 \\
\hline URL & ht t p: //hdl . handl e. net /10655/00012747 \\
\hline
\end{tabular}




\section{Analysis of higher harmonics on bidirectional heat pulse propagation experiment in helical and tokamak plasmas}

T. Kobayashi ${ }^{1,2}$, K. Ida ${ }^{1,2,3}$, S. Inagaki ${ }^{3,4}$, H. Tsuchiya ${ }^{1}$, N. Tamura ${ }^{1}$,

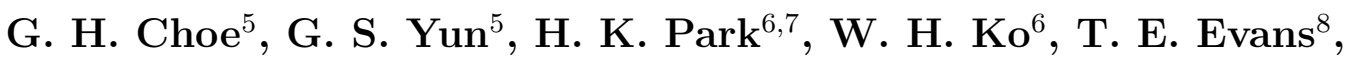

M. E. Austin ${ }^{9}$, M. W. Shafer ${ }^{10}$, M. Ono ${ }^{2}$, D. López-bruna ${ }^{11}$,

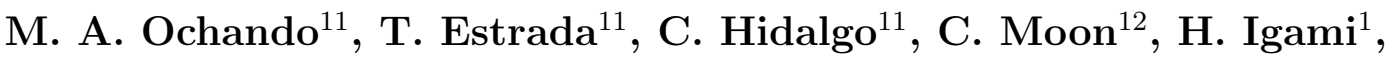

Y. Yoshimura ${ }^{1}$, T. Ii. Tsujimura ${ }^{1}$, S.-I. Itoh ${ }^{3,4}$, and K. Itoh ${ }^{1,3}$

${ }^{1}$ National Institute for Fusion Science, National Institutes of Natural Sciences, Japan

2 SOKENDAI (The Graduate University for Advanced Studies), Japan

${ }^{3}$ Research Center for Plasma Turbulence, Kyushu University, Japan

${ }^{4}$ Research Institute for Applied Mechanics, Kyushu University, Japan

${ }^{5}$ Pohang University of Science and Technology, Korea

${ }^{6}$ National Fusion Research Institute, Korea

${ }^{7}$ Ulsan National Institute of Science and Technology, Korea

${ }^{8}$ General Atomics, San Diego, USA

${ }^{9}$ University of Texas, USA

${ }^{10}$ Oak Ridge National Laboratory, USA

${ }^{11}$ Laboratorio Nacional de Fusión, CIEMAT, Madrid, Spain

12 Max-Planck-Institut für Plasmaphysik, Germany

E-mail: kobayashi.tatsuya@LHD.nifs.ac.jp

Abstract. In this contribution we analyze modulation electron cyclotron resonance heating (MECH) experiment and discuss higher harmonic frequency dependence of transport coefficients. 
We use the bidirectional heat pulse propagation method, in which both inward propagating heat pulse and outward propagating heat pulse are analyzed at a radial range, in order to distinguish frequency dependence of transport coefficients due to hysteresis from that due to other reasons, such as radially dependent transport coefficients, a finite damping term, or boundary effects. The method is applied to MECH experiments performed in various helical and tokamak devices, i.e., Large Helical Device (LHD), TJ-II, Korea Superconducting Tokamak Advanced Research (KSTAR), and Doublet III-D (DIII-D) with different plasma conditions. The frequency dependence of transport coefficients are clearly observed, showing a possibility of existence of transport hysteresis in flux-gradient relation.

\section{Introduction}

Predictive modeling of thermal transport in magnetically confined plasmas is mandatory to realize the fusion reactor. Recent studies have reported that electron thermal transport in the axially heated plasma cannot be modeled by classical diffusion transport model with a single scalar diffusive coefficient. There can exist critical gradient-type

nonlinearity $[1,2]$, inward pinch $[3,4,5,6]$, and ballistic transport events $[7,8,9]$. More recently, emergence of a hysteresis in flux-gradient relation was discovered $[10,11,12]$, involving rapid responses of turbulence intensity and turbulent transport against heating [11]. This mechanism can also explain a long standing mystery, that is, rapid increase of the electron thermal diffusivity in response to electron cyclotron resonance heating (ECH), found in Wendelstein 7-AS stellarator [13].

Modulation ECH (MECH) is widely used to provide perturbation in electron 
temperature, since its modulation frequency, input power, and deposition can be precisely controlled $[1,2,4,5,6,10,11,12,14,15,16]$. See also a review [17]. Hysteresis in flux-gradient relation can be assessed by MECH experiment. By analyzing not only fundamental MECH frequency but also its higher harmonics, i.e., the socalled higher harmonic method [18], possible violation from the classical diffusive transport picture can be discussed $[15,16]$. Amplitude radial decay rate of temperature perturbation is predicted to obey the square root of their frequencies in the case that linear local diffusion model is valid. The experimental observations $[15,16]$ showed a clear deviation from the linear local picture, where almost constant radial decay rates among the fundamental frequency and higher harmonics were observed. The constant slope provides transport coefficient that depends on frequency, showing a contradiction with linear local theory. Possible relation between the constant slope and transport hysteresis was also discussed [18].

In this contribution, we extend the higher harmonic method in order to discuss existence of hysteresis in flux-gradient relation. We also use both outward and inward propagating heat pulses to obtain the transport coefficients at a radial region, which we call the bidirectional heat pulse propagation method, in order to distinguish the frequency dependence of transport coefficients due to hysteresis from that due to other reasons, such as radially dependent transport coefficients, a finite damping term, or boundary effects. This method is applied to MECH experiments performed in various helical and tokamak devices, i.e., LHD, TJ-II, KSTAR, and DIII-D with different 
operation conditions. A clear dependence of transport coefficients on both propagation direction and higher harmonic frequency is obtained, showing a possibility of existence of transport hysteresis [19].

\section{Method}

On the one hand, if the classical local transport model works, obtained transport coefficients should be neither dependent on frequency nor on heat pulse propagating direction. On the other hand, according to a theory [19], difference between transport coefficients obtained from outward pulse and inward pulse becomes larger at higher frequency in the presence of hysteresis. By analyzing frequency dependence of diffusivity and convective velocity evaluated both from outward propagating pulse and from inward propagating pulse, we discuss possibility of existence of hysteresis in fluxgradient relation. We begin with one-dimensional heat transport equation for electron temperature perturbation $\delta T_{\mathrm{e}}$ in the cylindrical coordinate defined as

$$
\frac{\partial n \delta T_{\mathrm{e}}}{\partial t}+\frac{n \delta T_{\mathrm{e}}}{\tau}=-\frac{1}{r} \frac{\partial\left(r \delta q_{r}\right)}{\partial r}+\delta S
$$

where $\delta q_{r}$ is the perturbed radial heat flux and $\tau$ is the damping coefficient [17]. We analyze the region where the modulation heat source $\delta S$ is not deposited. The perturbed local heat flux is given by the classical local model as

$$
\delta q_{r}=-n \chi_{\mathrm{HP}} \frac{\partial \delta T_{\mathrm{e}}}{\partial r}+n V_{\mathrm{HP}} \delta T_{\mathrm{e}}
$$


where $\chi_{\mathrm{HP}}$ and $V_{\mathrm{HP}}$ are thermal diffusion coefficient and convective velocity, respectively, with respect to heat pulse. With the given heat flux, the transport equation becomes

$$
\frac{\partial T_{\mathrm{e}}}{\partial t}=\chi_{\mathrm{HP}} \frac{\partial^{2} T_{\mathrm{e}}}{\partial r^{2}}+\left(\frac{\chi_{\mathrm{HP}}}{r}-V_{\mathrm{HP}}\right) \frac{\partial T_{\mathrm{e}}}{\partial r}-\frac{V_{\mathrm{HP}}}{r} T_{\mathrm{e}}
$$

where $\tau$ is taken as infinity and $\chi_{\mathrm{HP}}$ and $V_{\mathrm{HP}}$ are treated as radially constant for simplicity. Density profile is also taken to be constant in radius. Influence of these simplification appears as a dummy frequency dependence in the transport coefficients. This point is discussed below. Considering electron temperature perturbation

$$
\delta T_{\mathrm{e}} \propto \exp \left[-i \omega t+i\left(k_{\mathrm{r}}+i k_{\mathrm{i}}\right) r\right]
$$

where $k_{\mathrm{r}}, k_{\mathrm{i}}$ and $\omega$ indicate the real part and the imaginary part of the radial wavenumber and the angular frequency, the transport coefficients can be obtained as

$$
\chi_{\mathrm{HP}}=\frac{1}{k_{\mathrm{r}}^{2}+k_{\mathrm{i}}^{2} \gamma^{2}} \frac{k_{\mathrm{i}} \gamma}{k_{\mathrm{r}}} \omega
$$

and

$$
V_{\mathrm{HP}}=\frac{k_{\mathrm{r}}^{2}-k_{\mathrm{i}}^{2} \gamma}{k_{\mathrm{r}}^{2}+k_{\mathrm{i}}^{2} \gamma^{2}} \frac{1}{k_{\mathrm{r}}} \omega
$$

The factor $\gamma \equiv 1-1 / k_{\mathrm{i}} r$ shows the cylindrical geometry effect that comes from the divergence operator. We have considered higher order corrections from cylindrical geometry, as well, by treating a slight radial variation of the wavenumber components with WKB approximation. See a dedicated article [20]. The wavenumbers $k_{\mathrm{r}}$ and $k_{\mathrm{i}}$ can be obtained from the Fourier analysis of $\delta T_{\mathrm{e}}$ combined with conditional averaging technique at odd number harmonic frequencies $f=f_{\mathrm{MECH}}, 3 f_{\mathrm{MECH}}, \ldots, m f_{\mathrm{MECH}}$, where $f_{\mathrm{MECH}}$ is the MECH frequency $(f=\omega / 2 \pi)$ and $m$ is odd integers. The local model 
predicts the wavenumbers being nearly proportional to $\sqrt{f}$, and thus $\chi_{\mathrm{HP}}$ and $V_{\mathrm{HP}}$ that do not depend on $f[15,16,20]$.

In general, when values of $n, \chi_{\mathrm{HP}}$ and $V_{\mathrm{HP}}$ have radial dependences, or when a finite value of damping term $\tau$ exists, obtained $\chi_{\mathrm{HP}}$ and $V_{\mathrm{HP}}$ naturally depend on frequency. In those cases, Eqs. (5) and (6) become

$$
\chi_{\mathrm{HP}}=\frac{1}{k_{\mathrm{r}}^{2}+k_{\mathrm{i}}^{2} \gamma^{2}}\left[\frac{k_{\mathrm{i}} \gamma}{k_{\mathrm{r}}} \omega-\frac{1}{\tau}+k_{\mathrm{i}}(\gamma-1) \chi_{\mathrm{HP}}^{\prime}-V_{\mathrm{HP}}^{\prime}\right]
$$

and

$$
V_{\mathrm{HP}}=\frac{1}{k_{\mathrm{r}}^{2}+k_{\mathrm{i}}^{2} \gamma^{2}}\left[\frac{k_{\mathrm{r}}^{2}-k_{\mathrm{i}}^{2} \gamma}{k_{\mathrm{r}}} \omega+k_{\mathrm{i}}(\gamma+1) \frac{1}{\tau}+\left(k_{\mathrm{i}}^{2}+k_{\mathrm{r}}^{2}\right) \chi_{\mathrm{HP}}^{\prime}+k_{\mathrm{i}}(\gamma+1) V_{\mathrm{HP}}^{\prime}\right] \text {, }
$$

respectively. Note that influence of the density profile is similar to that of radially dependent $\chi_{\mathrm{HP}}$ and $V_{\mathrm{HP}}$, thus we omit discussion here. An important fact is that the denominator $k_{\mathrm{r}}^{2}+k_{\mathrm{i}}^{2} \gamma^{2}$ increases as frequency increases, keeping the first terms constant, if the local model is valid. As a result, impacts of the other terms become smaller at higher frequencies. This asymptotic behavior is not identical for outward propagating pulse and inward propagating pulse. Therefore, the difference of the transport coefficients obtained from both pulses would decrease as frequency increases. Moreover, edge and core boundary effects show similar frequency dependence, i.e., asymptotic convergence to a true value at higher frequency. See Ref. [20]. When the frequency dependence is caused by the transport hysteresis, the difference of the transport coefficients obtained from inward pulse and outward pulse should increase at a higher frequency range [19]. The asymptotic behavior of higher harmonic terms is essential to discuss cause of the frequency dependence of the transport coefficients. 


\section{Experimental parameters}

Experiments are performed in steady state L-mode plasmas. The experimental conditions (major radius $R$, minor radius $a$, toroidal magnetic field strength $B$, plasma current $I_{\mathrm{p}}$, line averaged electron density $\bar{n}_{\mathrm{e}}$, and total heating power $P_{\text {tot }}$ ) are summarized in Table 1. MECH having a block-type waveform with fundamental frequency $f_{\mathrm{MECH}}$ and zero-to-peak amplitude $\delta P_{\mathrm{MECH}}$ is applied to investigate heat pulse propagation. Values of $f_{\mathrm{MECH}}$ and $\delta P_{\mathrm{MECH}}$ are given in Table 1 , as well. In order to show wide coverage of the method, various experimental conditions are used. By changing the MECH deposition location, outward and inward propagating electron temperature perturbations are generated in LHD and KSTAR, during which electron temperature profiles are maintained almost unchanged. In KSTAR, electron temperature perturbation is measured with an Electron Cyclotron Emission Imaging (ECEI) system, while conventional ECE systems are used in the other three devices.

\section{Results}

Figure 1 shows the radial profile of the $T_{\mathrm{e}}$ perturbation power and the phase difference for the case of outward propagating pulses. The Perturbation power profile and the phase difference profile are given not only for the fundamental frequency (black symbols) but also for the third harmonic frequency (red symbols) and the fifth harmonic frequency (blue symbols). Error bars show the statistical error obtained from variance of complex Fourier amplitude in each period. When the classical local transport model is valid, 
slopes in the perturbation power profile and the phase profile approximately change with $\sqrt{f}[20]$. Black dashed lines in the figures show least-squares fit for the perturbation power profile and the phase profile at the fundamental frequency. Red and blue dashed lines are predicted profiles having slopes $\sqrt{3}$ and $\sqrt{5}$ times larger than that of the fundamental frequency component. The results clearly show that the local model cannot explain power and phase profiles. Helical plasmas seem to have much faster phase propagation speed, i.e., relatively smaller slope, compared to tokamak plasmas.

Similar plots are given for inward propagating pulses, as shown in Fig. 2. Note that we do not have dedicated discharges for observing inward propagating heat pulse for DIII-D and TJ-II. For the case of DIII-D, inner side of the MECH deposition is analyzed. Dashed lines look to better fit the experiments with the inward propagating heat pulse, in particular for the case of KSTAR.

In order to discuss possible deviation from the classical local theory more quantitatively, we analyze diffusion coefficient and convective velocity for different harmonic frequencies and for both outward pulse and inward pulse. Slopes of Figs. 1 and 2 directly correspond to $k_{\mathrm{i}}$ and $k_{\mathrm{r}}$, i.e., $k_{\mathrm{i}}=A^{\prime} / A$ and $k_{\mathrm{r}}=\phi^{\prime}$, where $A$ and $\phi$ show the amplitude profile and the phase profile. Figure 3 shows frequency dependences of $k_{\mathrm{i}}$ and $k_{\mathrm{r}}$ for the different devices. The top side of the horizontal axis in Fig. 3 is labeled by normalized frequency by inverse of scaling confinement time, $\tau_{\mathrm{E}}^{-1}$, where ISS95 scaling [21] and ITER89-P scaling [22] are used for helical devices and tokamak devices. Wavenumbers are analyzed at $f=f_{\mathrm{MECH}}, 3 f_{\mathrm{MECH}}$, and $5 f_{\mathrm{MECH}}$. Black solid 
lines and red dashed lines show the predicted wavenumbers from the classical local theory using the transport coefficients obtained at the fundamental MECH frequency component [20]. In most cases, absolute value of $k_{\mathrm{i}}$ is larger than that of $k_{\mathrm{r}}$, regardless of the pulse propagation direction. In particular, helical devices have a much larger difference between $\left|k_{\mathrm{i}}\right|$ and $\left|k_{\mathrm{r}}\right|$, almost one order of magnitude difference. In addition, in the cases of LHD, TJ-II, and DIII-D, the real part of the wavenumber only weakly depends on the harmonic frequency and absolute values of $k_{\mathrm{r}}$ are significantly below the predicted values for higher harmonic components. This means that the phase of the heat pulse propagates much faster than the prediction from the classical local theory, which can provide a frequency dependence of the transport coefficients.

Using $k_{\mathrm{i}}$ and $k_{\mathrm{r}}$ in Fig. $3, \chi_{\mathrm{HP}}$ and $V_{\mathrm{HP}}$ are calculated for both outward and inward propagating heat pulses, as shown in Fig. 4, as a function of the harmonic frequency $f$ (only for outward pulse in the case of TJ-II). In the cases of LHD, TJ-II, and DIIID, obtained coefficients strongly depend on the frequency $f$, in which the local theory predicts constant coefficients against $f$. The KSTAR plasma shows a strong frequency dependence only in convective velocity. The difference between the transport coefficients obtained from outward pulses and inward pulses becomes larger as frequency increases. Therefore, this frequency dependence is not due to the radial dependence of $n, \chi_{\mathrm{HP}}$, and $V_{\mathrm{HP}}$, finite value of the damping term, nor boundary effects, as discussed in Sec. 2 . The polarity of convective velocities depends on the direction of heat pulse propagation. This is because the condition $k_{\mathrm{r}}^{2}-k_{\mathrm{i}}^{2} \gamma<0$ is always satisfied. Faster phase propagation 
may correspond to an immediate response of radial heat flux against turn on of heating $[13,11,18]$. The strong frequency dependence of transport coefficients in the present analysis indicates that the transient response during $\mathrm{MECH}$ cannot be explained in the classical local view of transport. These results showing a clear dependence of the transport coefficients on both propagation direction and higher harmonic frequency can be linked to existence of transport hysteresis [19]. It should be noted that authors in Refs $[2,6,14]$ claimed that frequency dependence of heat pulse transport coefficients is small enough to validate the classical local transport theory. Conditions that provide strong frequency dependence on the transport coefficients should be investigated to unveil the nonlocal characteristics of high temperature magnetically confined plasmas.

\section{Summary}

In this contribution, we extended higher harmonic method in order to discuss existence of hysteresis in flux-gradient relation. We used the bidirectional heat pulse propagation method in order to distinguish frequency dependence of the transport coefficients due to hysteresis from that due to other reasons, such as radially dependent transport coefficients, a finite damping term, or boundary effects. The method was applied to MECH experiments performed in various helical and tokamak devices, i.e., LHD, TJ-II, KSTAR, and DIII-D with different operation conditions. Results showed clear dependences of the transport coefficients on both propagation direction and higher harmonic frequency, showing a possibility of existence of transport hysteresis [19]. 
Within presented discharges, which include various sizes of helical and tokamak plasmas, violation from the classical local view of transport was universally observed.

\section{Acknowledgments}

We thank Profs. S. Sakakibara, A. Fujisawa, and Y. Kamada for strong support and continuous encouragement. This study is partly supported by a Grant-in-Aid for Scientific Research of JSPS, Japan (15H02155, 15K14282, 15H02335, and 16H02442) and by the collaboration programs of NIFS (NIFS13KOCT001) and the RIAM of Kyushu University, and by Asada Science Foundation. 


\section{Reference}

[1] F Ryter, G Tardini, F De Luca, H-U Fahrbach, F Imbeaux, A Jacchia, KK Kirov, F Leuterer,

P Mantica, AG Peeters, et al 2003 Nucl. Fusion 431396

[2] J C DeBoo, C C Petty, A E White, K H Burrell, E J Doyle, J C Hillesheim, C Holland, G R McKee, T L Rhodes, L Schmitz, et al 2012 Phys. Plasmas 19082518

[3] T C Luce, C C Petty, and J C M De Haas 1992 Phys. Rev. Lett. 6852

[4] P Mantica, G Gorini, G M D Hogeweij, N J Lopes Cardozo, and A M R Schilham 2000 Phys. Rev. Lett. 854534

[5] P Mantica, F Ryter, C Capuano, H U Fahrbach, F Leuterer, W Suttrop, Jan Weiland, and ASDEX-Upgrade Team 2006 Plasma Phys. Control. Fusion 48385

[6] S D Song, X L Zou, G Giruzzi, W W Xiao, X T Ding, B J Ding, J L Ségui, D Elbèze, F Clairet, C Fenzi, et al 2012 Nucl. Fusion 52033006

[7] E D Fredrickson, K McGuire, A Cavallo, R Budny, A Janos, D Monticello, Y Nagayama, W Park, G Taylor, and M C Zarnstorff 1990 Phys. Rev. Lett. 65 2869-2872

[8] M W Kissick, E D Fredrickson, J D Callen, C E Bush, Z. Chang, P C Efthimion, R A Hulse, D K Mansfield, H K Park, J F Schivell, et al 1994 Nucl. Fusion 34349

[9] K W Gentle, W L Rowan, R V Bravenec, G Cima, T P Crowley, H Gasquet, G A Hallock, J Heard, A Ouroua, P E Phillips, D W Ross, P M Schoch, and C Watts 1995 Phys. Rev. Lett. $743620-3623$

[10] K W Gentle, M E Austin, J C DeBoo, T C Luce, and C C Petty 2006 Phys. Plasmas 13012311

[11] S Inagaki, T Tokuzawa, N Tamura, S-I Itoh, T Kobayashi, K Ida, T Shimozuma, S Kubo, K Tanaka, T Ido, et al 2013 Nucl. Fusion 53113006

[12] K Ida, Z Shi, HJ Sun, S Inagaki, K Kamiya, JE Rice, N Tamura, PH Diamond, G Dif-Pradalier, XL Zou, et al 2015 Nucl. Fusion 55013022

[13] U Stroth, L Giannone, HJ Hartfuss, ECH Group, et al 1996 Plasma Phys. Control. Fusion 38611 
[14] L Giannone, V Erckmann, U Gasparino, HJ Hartfuss, G Kuhner, H Maassberg, U Stroth, and M Tutter 1992 Nucl. Fusion 321985

[15] S Inagaki, S-I Itoh, K Itoh, N Kasuya, T Kobayashi, A Fujisawa, T Tokuzawa, K Ida, S Kubo, T Shimozuma, et al 2013 Plasma Fusion Res. 81202173

[16] S Inagaki, S-I Itoh, K Itoh, K Ida, D López-Bruna, M A Ochand, T Estrada, B Ph van Milligen, C Hidalgo, and N Kasuya 2014 Plasma Fusion Res. 91202052

[17] N J Lopes Cardozo 1995 Plasma Phys. Control. Fusion 37799

[18] K Itoh, S-I Itoh, S Inagaki, N Kasuya, and A Fujisawa 2015 J. Phys. Soc. Jpn. 85014501

[19] K Itoh 2016 Proc. 26th IAEA Fusion Energy Conf. (Kyoto, Japan) (Vienna: IAEA) pages OV/P8

[20] T Kobayashi, K Itoh, K Ida, S Inagaki, and S-I Itoh 2017 submitted to J. Phys. Soc. Jpn.

[21] U Stroth, M Murakami, R A Dory, H Yamada, S Okamura, F Sano, and T Obiki 1996 Nucl. Fusion 361063

[22] P N Yushmanov, T Takizuka, K S Riedel, O J W F Kardaun, J G Cordey, S M Kaye, and D E Post 1990 Nucl. Fusion 301999 
Table 1. Experimental conditions

\begin{tabular}{lcccccccc}
\hline Device & $R[\mathrm{~m}]$ & $a[\mathrm{~m}]$ & $B[\mathrm{~T}]$ & $I_{\mathrm{p}}[\mathrm{MA}]$ & $\bar{n}_{\mathrm{e}}\left[10^{19} \mathrm{~m}^{-3}\right]$ & $P_{\text {tot }}[\mathrm{MW}]$ & $\delta P_{\mathrm{MECH}}[\mathrm{MW}]$ & $f_{\mathrm{MECH}}[\mathrm{Hz}]$ \\
\hline LHD & 3.6 & 0.6 & 2.75 & - & 1.3 & 5.2 & 0.38 & 18 \\
TJ-II & 1.5 & 0.2 & 1 & - & 0.5 & 0.275 & 0.025 & 180 \\
KSTAR & 1.8 & 0.5 & 2.9 & 0.5 & 2 & 1.78 & 0.25 & 180 \\
DIII-D & 1.7 & 0.6 & 1.97 & 1.29 & 3.35 & 3.25 & 1.35 & 50 \\
\hline
\end{tabular}



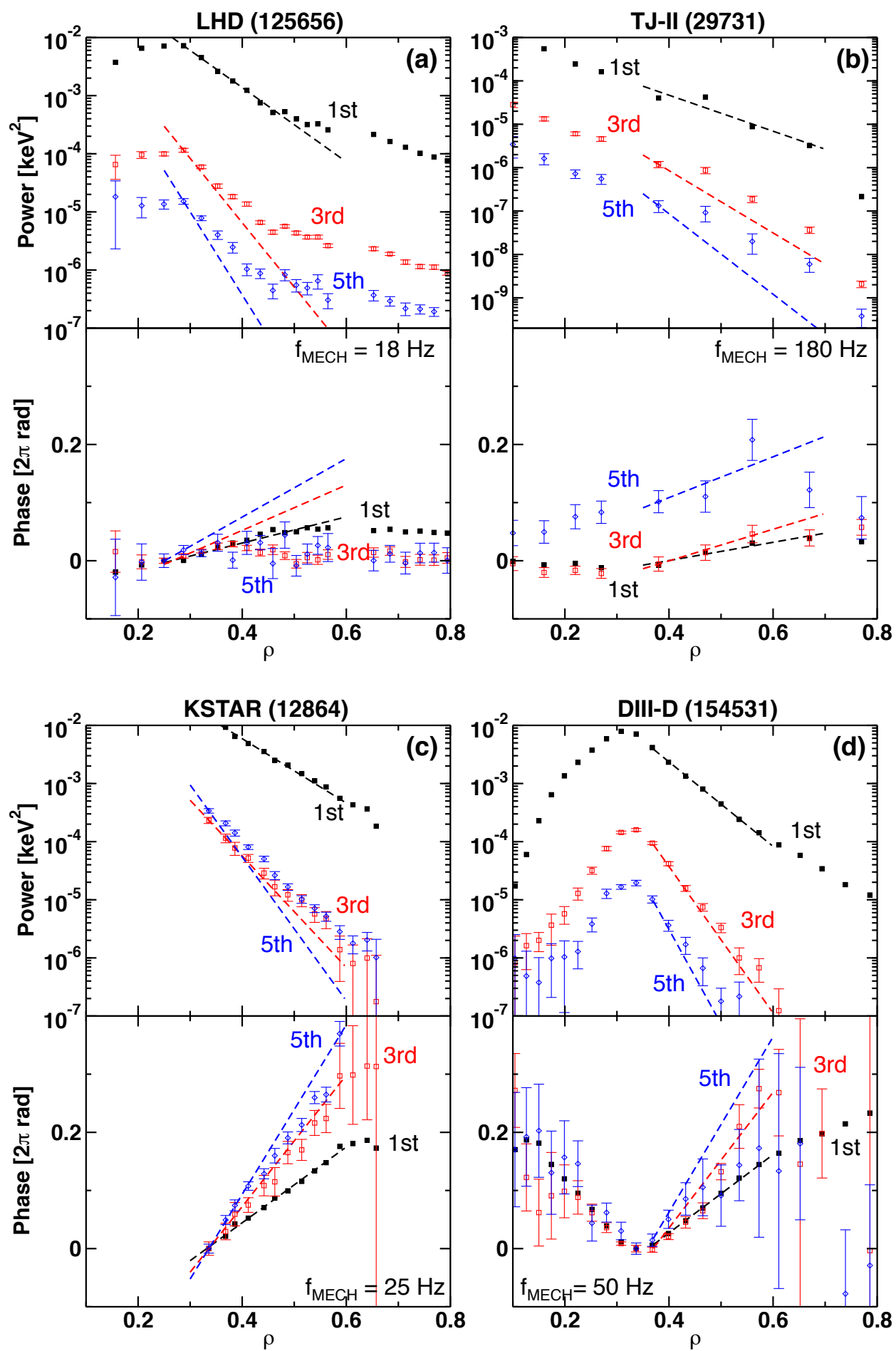

Figure 1. Radial profiles of electron temperature perturbation (top) and phase difference (bottom) for (a) LHD, (b) TJ-II, (c) KSTAR, and (d) DIII-D for analyzing outward propagating pulses. Dashed lines in the figure show the prediction from pure diffusion theory, in which thermal diffusivity is obtained from the fundamental mode. 

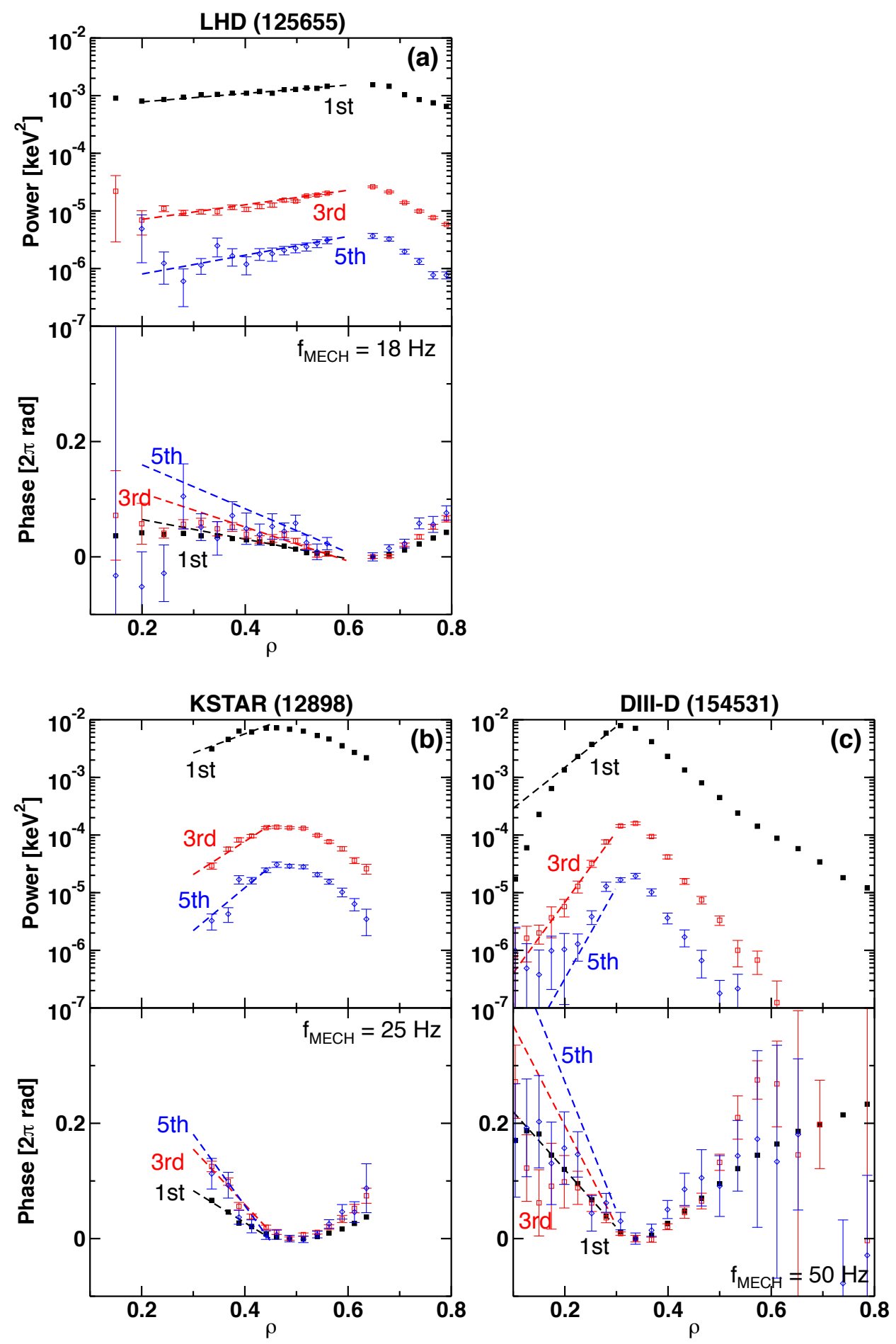

Figure 2. Radial profiles of electron temperature perturbation (top) and phase difference (bottom) for (a) LHD, (b) KSTAR, and (c) DIII-D for analyzing inward propagating pulses. Dashed lines in the figure show the prediction from pure diffusion theory, in which thermal diffusivity is obtained from the fundamental mode. 

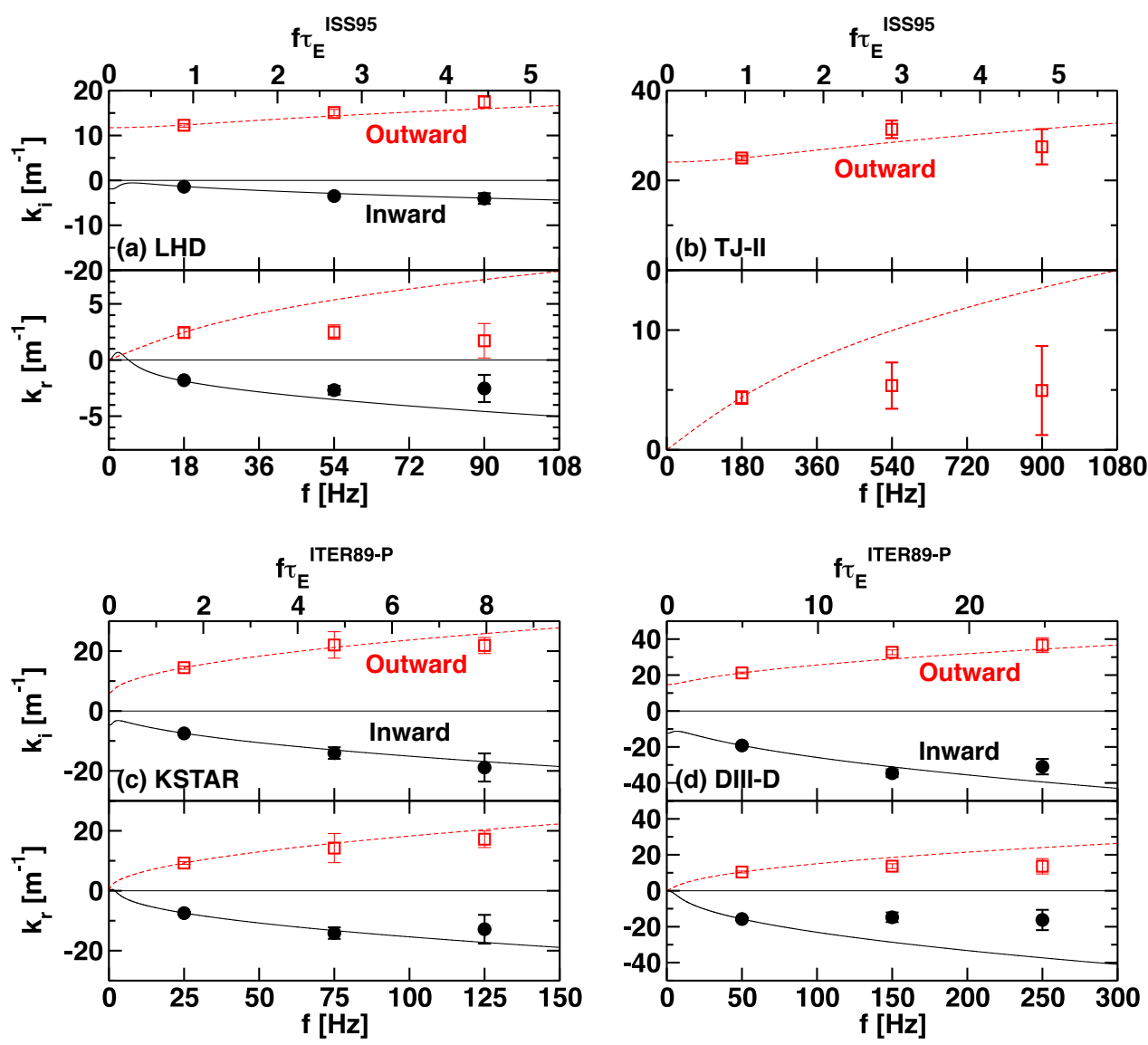

Figure 3. Imaginary part of wavenumber $k_{\mathrm{i}}$ (top) and real part of wavenumber $k_{\mathrm{r}}$ (bottom) as a function of the modulation harmonic frequency. Black solid lines and red dashed lines show predicted wavenumbers from the classical local theory with the transport coefficients obtained at the fundamental MECH frequency component. 

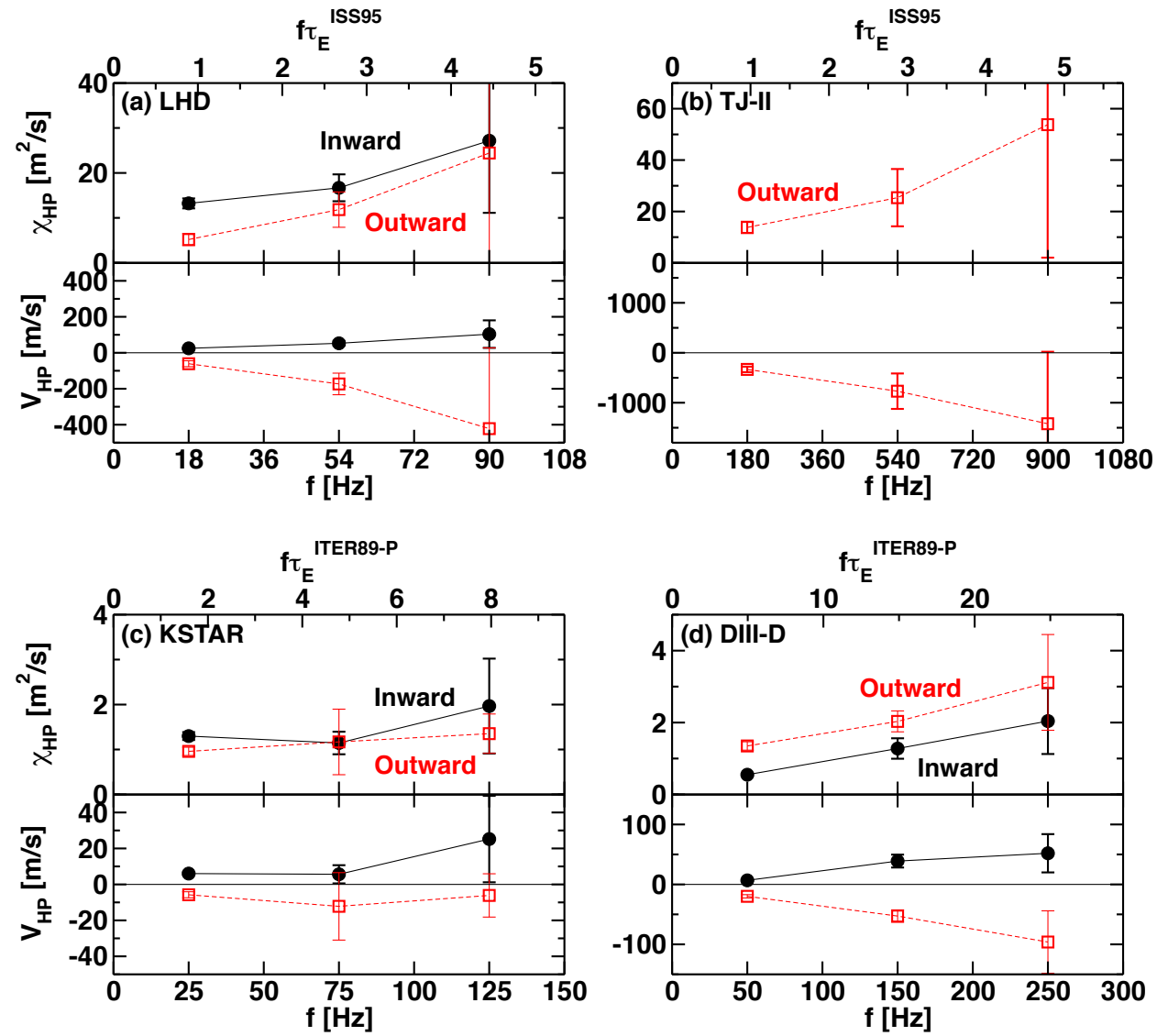

Figure 4. Thermal diffusivity (top) and convective velocity (bottom) as a function of the modulation harmonic frequency. 